\title{
DESENVOLVIMENTO DE METODOLOGIA DE REVELAÇÃO DE IMPRESSÃO DIGITAL LATENTE COM CHALCONAS
}

\author{
Jaqueline D. Balsan ${ }^{\mathrm{a}}$, Bruno N. Rosa ${ }^{\mathrm{a}}$, Claudio M. P. Pereira ${ }^{\mathrm{a}, \mathrm{b}}$ e Clarissa M. M. Santos ${ }^{\mathrm{a}, *, \odot}$ \\ ${ }^{a}$ Centro de Ciências Químicas, Farmacêuticas e de Alimentos, Universidade Federal de Pelotas, Campus Capão do Leão, 96160-000 \\ Pelotas - RS, Brasil \\ 'Instituto Nacional de Ciência e Tecnologia Forense, 96010-900 Pelotas - RS, Brasil
}

Recebido em 12/02/2019; aceito em 10/07/2019; publicado na web em 26/08/2019

\begin{abstract}
DEVELOPMENT OF METHODOLOGY OF LATENT FINGERPRINT REVELATION WITH CHALCONES. Fingerprints are unique for each individual contributing to human identification in forensic cases. Visualization of latent fingerprints usually involves the use of a chemical substance that creates a contrast between fingerprint residues and the surface on which the print was deposited. Commercial powders used as fingerprint developers by papilloscopy have relatively high costs due to their importation and chemical composition. (E-1,3-diphenylprop-2-en-1-one, $E$-1-phenyl-3-(4-methylphenyl))prop-2-en-1-one but only one compound was highlighted, E-3-(3-nitrophenyl)-1-phenylprop-2-em-1-one). The composite was selected for its suitable properties (adhesion and texture) of visualization of the digital, providing the obtaining of clear revelations with identification of minutiae and singular points of the fingerprints. Identification, magnetic resonance, granulometry and contact surface evaluation with chalcones such as sebaceous and natural deposition with development in different materials such as plastic, metal and glass were applied for the application of fingerprint development. The compound was tested in eight volunteers regardless of gender and age, presenting satisfactory results for fingerprint development on glass and metal surfaces.
\end{abstract}

Keywords: chalcones; latent fingerprint; developer powder.

\section{INTRODUÇÃO}

Impressões digitais latentes podem ser descritas como vestígios deixados no local do crime, podendo ser visíveis ou não. ${ }^{1}$ As impressões podem ser visíveis ou não: impressões digitais latentes visíveis mostram detalhes identificáveis das papilas dos dedos, quando contaminados por substâncias, tais como o sangue, tinta, gordura ou sujeira que naturalmente deixam um contraste ou são produzidas quando impressões digitais são pressionadas contra superfícies plásticas. ${ }^{2}$

As impressões digitais latentes ("escondidas") não são visíveis a olho nu- e consistem, substancialmente, apenas de secreções naturais da pele humana e, tais impressões, necessitam de tratamento para se tornarem visíveis. Esse tratamento é denominado de "revelação de impressão digital latente" sendo necessário utilizar alguns processos químicos ou físicos, nos quais ao interagir com as secreções da pele faz com que a impressão digital latente apresente contraste. A escolha de metodologia adequada para a revelação é de extrema importância para conhecimento da constituição da impressão digital. ${ }^{3}$

O conjunto de procedimentos e metodologias adotados no local de crime, disciplinando os exames periciais a serem realizados nos objetos que foram manipulados em outros materiais e mesmo na vítima é denominado Revelação de Impressões Papilares. Após iniciar a revelação de impressões papilares na cena do crime são obtidos arquivos com fragmentos revelados, os quais eram comparados com os suspeitos. ${ }^{4}$ A importância da coleta deste tipo de vestígio é devido ao fato de cada ser humano possuir um conjunto único de impressões digitais que acompanham desde a gestação até sua morte sem apresentar mudanças significativas. ${ }^{5-7}$

A identificação de impressões digitais é uma das mais importantes formas de investigação criminal devido a duas características: a sua unicidade (impressão individual única para cada indivíduo) e a sua imutabilidade (sem mudanças significativas). Assim, as cristas de

*e-mail: clafarm_mm@yahoo.com.br fricção apresentam as impressões digitais formadas dentro do útero materno e se desenvolvem proporcionalmente ao desenvolvimento do bebê.

Além disso, as impressões digitais de uma pessoa não mudam ao longo do tempo, o que pode ocorrer é a cicatrização permanente (ferimento na ponta dos dedos e posterior cicatrização), sendo a única maneira de uma impressão digital ser alterada. ${ }^{8,9}$

Quando da realização dos exames periciais voltados a revelação das impressões papilares, seja no local de crime ou em laboratório, a aplicação de qualquer técnica deve ser precedida de confiabilidade de marcas de dedos latentes como evidência em processos judiciais. Consequentemente, a papiloscopia possui características simples e possibilita a praticabilidade na coleta de impressões papilares, sendo considerado um método de baixo custo, rápido, eficaz e que permite o arquivamento das impressões para posteriores classificações e auxílio na identificação humana. ${ }^{10-15}$

No entanto, devido aos pós comerciais apresentarem custos relativamente altos (devido à importação dos produtos), toxicidade e também dificuldade de visibilidade das minúcias e pontos singulares das impressões digitais, alguns estudos têm sido feitos com substâncias naturais para o desenvolvimento de novos pós reveladores de impressão digital mais acessíveis quanto a custo e baixa toxicidade.

Dessa forma, as chalconas, sendo produtos naturais e pertencendo à família dos flavonoides, pode também ser sintetizadas usando protocolos sintéticos orgânicos convencionais e favorecendo o processo de síntese de química limpa e atendendo aos requisitos de qualidade de revelação de impressão digital. As chalconas substituídas são de particular interesse para vários estudos devido à sua ampla gama de atividades biológicas, sendo a estrutura básica mostrada na Figura 1.

De acordo com a Figura 1, as chalconas ou 1,3-difenil-2propen1 -ona são definidas como cetonas $\alpha$ - $\beta$-insaturadas, onde tem uma ligação tanto na carbonila quanto na porção olefínica de grupamentos aromáticos comumente designados como anel $\mathrm{A}$, proveniente da acetofenona e o anel B do aldeído. 
<smiles>O=C(C=Cc1ccccc1)c1ccccc1</smiles>

Figura 1. Estrutura básica da chalcona

Existem várias técnicas para sua síntese, sempre visando variações estruturais e melhores rendimentos das reações. ${ }^{16}$ Dentre as atividades apresentadas pelas chalconas, podemos destacar: anti-inflamatória, ${ }^{17}$ antifúngica, antibacteriana, ${ }^{16}$ antimalárica, ${ }^{18}$ antileishmania, antitubercular $^{19}$ e antioxidante. ${ }^{20}$

Neste estudo foi proposto o desenvolvimento de metodologia de revelação de impressão digital latente com aplicação de diferentes chalconas ( $E$-1,3-difenilprop-2-en-1-ona, $E$-1-fenil-3(4-metilfenil) prop-2-en-1-ona, mas apenas o $E$-3-(3-nitrofenil)-1-fenil prop-2-en1-ona apresentou eficiência de adesão superior aos demais compostos pelas suas propriedades adequadas de adesão e textura, favorecendo a revelação de impressão digital latente com identificação de minúcias e pontos singulares das impressões digitais.

Sendo assim, o objetivo deste trabalho foi avaliar a aplicação de chalconas no desenvolvimento de metodologia de revelação de impressão digital latente em diferentes superfícies de contato auxiliando na acessibilidade de pós, baixo custo e qualidade de revelação de impressão digital confirmando a objetividade e confiabilidade da papiloscopia como método seguro e eficaz na identificação humana.

\section{PARTE EXPERIMENTAL}

\section{Síntese do composto}

Os compostos foram sintetizados ${ }^{21}$ através da reação entre o aldeído 3-nitrobenzaldeído (a) (5 mmol) com acetofenona (b) (5 mmol) em $8 \mathrm{~mL}$ de solução etanol/água (2:1) de hidróxido de sódio $(6 \mathrm{mmol})$. Essa solução ficou sob agitação durante $4 \mathrm{~h}$, sendo necessário resfriamento da reação. Após o tempo indicado, o sólido obtido E-3-(3-nitrofenil)-1-fenil prop-2-en-1-ona (c) foi filtrado sob vácuo e recristalizado com hexano/acetato de etila a fim de se obter os produtos puros, conforme mostrado nas Figura 2.

\section{Ressonância magnética nuclear (RMN)}

Os espectros de RMN ${ }^{1} \mathrm{H}$ e ${ }^{13} \mathrm{C}$ foram obtidos em um espectrômetro Bruker Ascend 400, na frequência de 400 MHz (Laboratório de Ressonância Magnética Nuclear - UFPel). Os desvios químicos ( $\delta)$ estão relacionados em parte por milhão ( $\mathrm{ppm}$ ) em relação ao padrão interno (TMS, usado como padrão interno para os espectros de ${ }^{1} \mathrm{H}$ NMR e $\mathrm{CDCl}_{3}$ para os espectros de ${ }^{13} \mathrm{C} \mathrm{NMR}$ ), colocando parênteses a multiplicidade ( $\mathrm{s}=$ singlet, $\mathrm{d}=$ doublet, $\mathrm{dd}=$ doublet, $\mathrm{t}=$ tripleto, quin $=$ quinteto, sex $=$ sexteto e $\mathrm{m}=$ multipleto), o número de hidrogênios deduzidos da integral relativa e da constante de acoplamento(J) expresso em Hertz (Hz).<smiles>O=Cc1cccc([N+](=O)[O-])c1</smiles>

a<smiles>CC(=O)c1ccccc1</smiles>

b

\section{Materiais e reagentes}

Os reagentes utilizados foram a acetofenona, hexano e acetato de etila sendo de procedência Merck ${ }^{\circledR}$. Etanol, hidróxido de sódio e os aldeídos substituídos utilizados nas sínteses são de procedência Aldrich ${ }^{\circledR}$. O nitrogênio líquido utilizado foi procedência White Martins ${ }^{\circledR}$ (Brasil).

Os materiais utilizados foram pincéis específicos de procedência Sirchie ${ }^{\circledR}$ sob os códigos 132LBW e CFB100. Câmera digital semiprofissional Canon EOS Rebel T6 foi utilizada para a obtenção das imagens e o pó padrão "Gold Metallic N BPP8916 Hi-Fi Pó" de procedência Sirchie ${ }^{\circledR}$ foi adquirido da Forensics Brazil.

Os pós reveladores de chalcona foram moídos em moinho criogênico (Spex - Modelo 6700), no qual os pós foram colocados em tubos individualizados e imersos em nitrogênio líquido para a obtenção de um material com granulometria adequada, permitindo perfeita homogeneização.

\section{Cromatografia a gás acoplada a espectrometria de massas (GC-MS)}

Para identificação dos compostos sintetizados foi utilizado um cromatógrafo à gás acoplado ao espectrômetro de massas (GC-MS) da marca Shimadzu, modelo GC-MS-QP 2010SE, equipado com injetor automático AOC-20i.

\section{Visualização microscópica das impressões digitais}

A caracterização das amostras para avaliação de chalconas como potencial revelador de impressão digital foi feita por Microscopia Eletrônica de Varredura (MEV) utilizando um equipamento Shimadzu, modelo SSX-550 Superscan.

As superfícies foram limpas para remover quaisquer impurezas superficiais. Foi preparado o tamanho padrão de cada substrato $(10 \mathrm{~mm} \times 10 \mathrm{~mm})$, no qual as chalconas e o revelador padrão de ouro foram testados separadamente. Os substratos (vidro e plástico) foram montados nas pontas com fita de carbono, metalizada com ouro (espessura de $20 \mathrm{~nm}$ ) e analisadas usando aceleração de feixe entre $5 \mathrm{kV}$ e $15 \mathrm{kV}$.

\section{Análise do tamanho de partículas}

As análises de tamanho de partículas foram realizadas usando um difratômetro a laser - isopoCILAS 1064. Para a análise, as amostras foram dispersas por ultra-som por 5 minutos com água como agente dispersante.

\section{RESULTADOS E DISCUSSÃO}

\section{Desenvolvimento de metodologia de revelação de impressão digital}

\section{Visualização microscópica das impressões digitais}

$\mathrm{Na}$ revelação de impressões digitais latentes aplica-se uma substância química ou física que revelam a deposição de resíduos papilares

Condições de reação: $i$ : NaOH; EtOH; EtOH, sob resfriamento; $4 \mathrm{~h}$

Figura 2. Reação-da síntese da chalcona. a: benzaldeído, b: acetofenona e c:chalcona 
que posteriormente revelação na superfície na qual a impressão foi depositada. Para a visualização microscópica foi utilizado o MEV. Foi preparado o tamanho padrão para a deposição de cada substrato (10 mm x $10 \mathrm{~mm})$, no qual as chalconas e o revelador padrão foram testados separadamente.

Os substratos (vidro e plástico) foram montados nas pontas com fita de carbono, metalizada com ouro e analisadas usando aceleração de feixe entre $5 \mathrm{kV}$ e $15 \mathrm{kV}$. Na Figura 3 são mostradas as imagens (a), (b), (c) e (d) possuem ampliações de $50 \times$ e $100 \times$ proporcionando uma melhor visualização das minucias da impressão digital latente utilizando amostra sintetizada de E-3-(3-nitrofenil)1-fenil prop-2-en-1-ona.
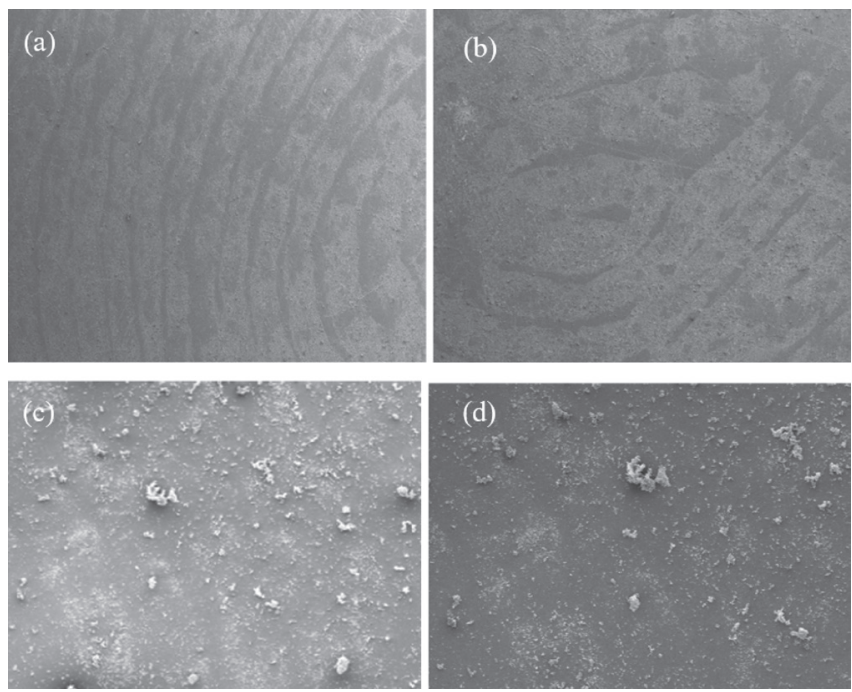

Figura 3. (a) Amostra sintetizada de E-3-(3-nitrofenil)-1-fenil prop-2-en1-ona depositada em substrato de plástico ampliada 50x. (b) idem ao " $a$ ", porém ampliada 100x. (c) Amostra sintetizada de E-3-(3-nitrofenil)-1-fenil prop-2-en-1-ona depositada em substrato de vidro ampliada 50x . (d) idem ao "a", porém ampliada $100 x$

De acordo com a Figura 3 obtida por imagens da MEV, nas imagens (a), (b), (c) e (d) foi possível observar uma interação adequada da chalcona com as superfícies de vidro e plástico. Assim, nota-se que o pó desenvolvido aderiu as impressões digitais mostrando linhas preenchidas e também alguns poros presentes nas impressões digitais, podendo ser visualizados em detalhes nas imagens. Neste estudo também foi utilizado para avaliação o pó revelador padrão (pó "Hi-Fi” dourado metálico) obtendo boa revelação digital e visualização das minúcias.

\section{Análise do tamanho de partículas}

De acordo com os histogramas da chalcona sintetizada e do pó revelador padrão mostrados na Figura 4 foi possível informar que ambas as análises apresentaram um ombro e um pico no gráfico de distribuição de densidade, indicando grau de heterogeneidade no tamanho das partículas.

Além disso, foi observada uma grande similaridade no tamanho das partículas da chalcona sintetizada e do padrão, de modo que os dois estão na maior parte na mesma escala e a morfologia dos grãos do pó revelador padrão e da chalcona sintetizada, respectivamente. Formas semelhantes foram observadas em diferentes regiões amostrais.

Na Tabela 1 são mostrados os valores de frequência das distribuições D10, D50 e D100, de acordo com os diâmetros das partículas nas faixas abaixo de $10 \%, 50 \%$ e $100 \%$ da curva de distribuição de tamanho, bem como o diâmetro médio chalcona sintetizada e do pó revelador padrão.

No histograma da distribuição de tamanhos pela técnica de granulometria a laser para a chalcona sintetizada e para o pó padrão revelou que o diâmetro médio das partículas foi de $114,36 \mu \mathrm{m}$ e $116,66 \mu \mathrm{m}$, respectivamente.

De acordo com o observado na Tabela 1, a chalcona sintetizada apresentou resultado adequado quanto ao tamanho de partícula quando comparada à amostra padrão, favorecendo a espalhabilidade do pó nas superfícies avaliadas, indicando seu potencial como revelador de impressões digitais latentes.

\section{Avaliação de chalcona como revelador de impressões digitais latentes}

Os compostos de chalconas foram testados como agentes reveladores de impressões digitais latentes de forma isolada. E para avaliação da adesão de digitais nas superfícies de contato com a aplicação do pó revelador de chalconas foram utilizados objetos com diferentes classificações de superfícies para a deposição das impressões digitais. Os pós reveladores de chalcona foram moídos em moinho criogênico, obtendo-se um material granulometria adequada e permitindo perfeita homogeneização.

Logo em seguida, as mãos dos doadores das digitais, foram lavadas com água potável e sabão neutro e, após 30 minutos, a impressão digital foi recolhida nas superfícies avaliadas. Após 24 h da deposição,
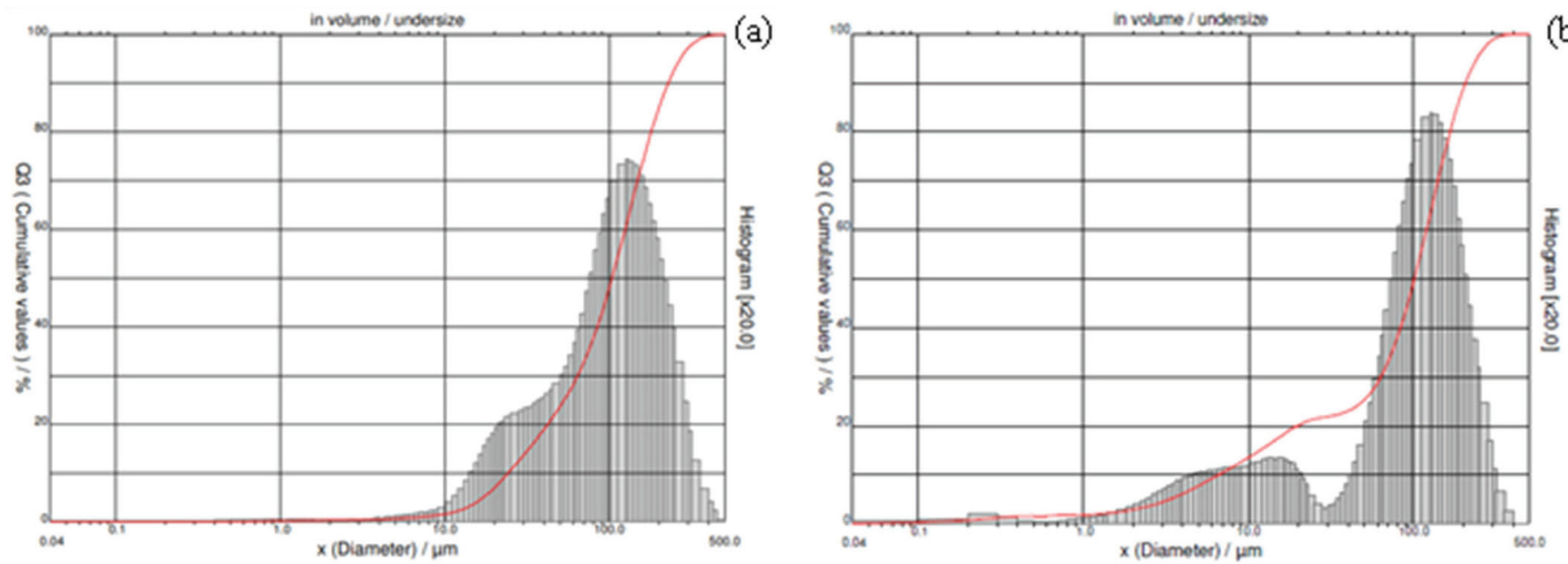

Figura 4. Histograma obtido da dispersão do tamanho de partículas. (a) Pó revelador comercial padrão (pó "Hi-Fi” dourado metálico) e (b) composto de chalcona (E-3-(3-nitrofenil)-1-fenil prop-2-en-1-ona) 
Tabela 1. Tamanho de partícula dos compostos chalcona e padrão

\begin{tabular}{ccccc}
\hline Composto & D 10\% $(\mu \mathrm{m})$ & D 50\% $(\mu \mathrm{m})$ & D 100\% $(\mu \mathrm{m})$ & Diâmetro médio \\
\hline Chalcona & 6,60 & 100,86 & 205,64 & 114,36 \\
Padrão & 24,19 & 103,50 & 227,36 & 116,66 \\
\hline
\end{tabular}

a revelação foi realizada com o auxílio de pincéis específicos para a aplicação.

As revelações foram fotografadas com câmera semiprofissional Canon EOS Rebel T6 e para fins de comparação, utilizou-se pó revelador comercial padrão (pó "Hi-Fi" dourado Metálico). Para avaliar a reprodutibilidade da amostra, essa foi comparada com o revelador padrão, sendo possível evidenciar seu potencial revelador de impressão digital latente mostrado na Figura 5.

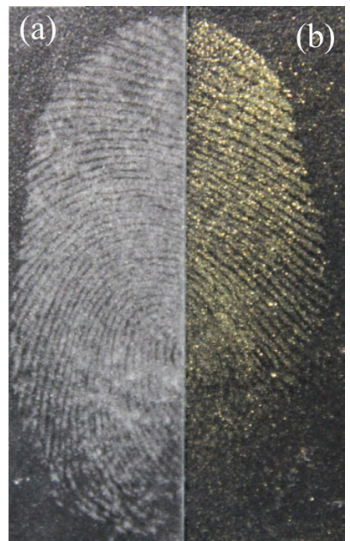

Figura 5. Imagem obtida por fotografia comparando a revelação de impressão digital da chalcona padrão com a chalcona sintetizada. (a) E-3-(3-nitrofenil)-1-fenil prop-2-en-1-ona e (b) pó revelador comercial padrão

Conforme mostrado na Figura 5 (a) e (b), foi possível observar o potencial revelador do pó $E$-3-(3-nitrofenil)-1-fenil prop-2-en-1-ona com desenho morfométrico, adequado dos sulcos das pontas dos dedos pelo atrito com a superfície e, também, uma alta sensibilidade para revelação de impressões digitais latentes comparando com o pó revelador comercial padrão. Além disso, o composto foi testado em diferentes superfícies para avaliar seu desempenho para aplicação deste pó revelador proposto.

\section{Aplicação dos compostos de chalconas de revelação de impressão digital latente}

\section{Deposição de impressões digitais latentes}

Os análogos sintéticos da Figura 1 da chalcona foram testados como agentes reveladores para desenvolvimento de revelação de impressões digitais latentes. Para preparar o pó, os análogos foram macerados com nitrogênio líquido e foram testados três compostos análogos da chalcona, 1,3-difenilprop-2-en-1-ona, 1-fenil-3(4-metilfenil)prop-2-en-1-ona, mas apenas um composto o $E$-3-(3-nitrofenil)1-fenil prop-2-en-1-ona, apresentou potencial revelador e, após essa prévia seleção, foi desenvolvida a metodologia de análise de revelação de impressão digital.

Dessa forma, foi procedida a coleta das impressões digitais latentes que foram depositadas por 8 voluntários que pressionaram as pontas dos dedos em lâminas de vidro limpas ou na superfície limpa de plástico.

Os doadores não foram selecionados com base em critérios de seleção especiais. Para uma deposição natural (deposição écrina, pois possui menos resíduos de gordura) de impressões digitais, as mãos foram lavadas com sabão neutro e água e continuaram fazendo suas atividades habituais por um período de 30 minutos. Para uma deposição sebácea, o doador esfregou o polegar em áreas do rosto, como a testa e o nariz (regiões de maior quantidade de glândulas sebáceas e acúmulo de oleosidade) e, posteriormente esfregou as pontas dos dedos para enriquecer a impressão com componentes oleosos.

Ao colocar as impressões digitais, a pressão foi subjetivamente firme (exercendo pressão média) e o tempo de contato ficou entre 3 e 5 segundos (tempo médio de contato do dedo). Após a deposição, as impressões digitais latentes foram mantidas durante 24 horas à temperatura ambiente para simular um trabalho real, e seu desenvolvimento foi realizado pelo método do pó (método físico) utilizando pincéis específicos (132LBW e CFB100 da Sirchie ${ }^{\circledR}$ ) para o desenvolvimento e limpeza do excesso de pó. O pó de análogo de chalcona foi comparado com pó de ouro comercial (Gold Metallic $N^{\circ}$ BPP8916 Hi-Fi Pó da Sirchie ${ }^{\circledR}$ adquirido da Forensics Brazil) apresentando cor semelhante às substâncias testadas. As fotografias foram feitas com uma câmera semiprofissional modelo Canon EOS Rebel T6.

Os testes feitos em superfície de plástico com chalcona sintetizada como pó revelador de impressão digital latente não apresentaram revelação adequada, por isso a partir daqui serão mostrados os testes feitos em superfície de vidro. Na Figura 6, as impressões reveladas com a chalcona sintetizada foram obtidas após 24 h com deposição sebácea por oito doadores aleatórios, sem distinção de sexo e idade.

Além disso, foram feitos também testes de revelação de impressão digital com a chalcona sintetizada após 24 h de deposição, mas com deposição écrina/natural, ou seja, as mãos foram lavadas $30 \mathrm{~min}$ antes da deposição por oito doações aleatórios, sem distinção de sexo e idade.

Dessa forma, como mostrado na Figura 6, houve visualização adequada das impressões digitais latentes, sendo possível fazer uma prévia classificação a "olho nu" dos principais tipos de impressões digitais encontrados, bem como apresentando algumas minúcias de forma de acordo com o Sistema Vucetich de Classificação de Impressões Digitais. Com base nos resultados obtidos, considerando a análise visual e morfológica das substâncias sintéticas testadas, o composto foi adequado para o desenvolvimento de impressões digitais latentes, sendo este um estudo inicial que propõe novas chalconas sintéticas como agentes de reveladores de impressões digitais latentes.

\section{CONCLUSÃO}

Conforme foi observado pelos resultados obtidos neste trabalho, os compostos sintetizados análogos da chalcona foram eficazes para o estudo proposto, mostrando adequada a chalcona com substituinte nitro. No entanto, o composto $E$-3-(3-nitrofenil)-1-fenil prop-2-en1-ona permitiu o desenvolvimento de impressões digitais em superfícies de vidro, apresentando desenho morfométrico dos sulcos de atrito adequado e de alta sensibilidade, principalmente nas marcas de dedos sebáceos.

Cabe salientar que o estudo proposto foi satisfatório, pois o composto sintetizado de chalcona facilita a aplicação de forma acessível, baixo custo, qualidade de revelação de impressão digital, além da síntese do composto apresentar redução de resíduos ao ambiente e provavelmente de exposição tóxica aos peritos pelo uso em grande quantidade desses pós numa cena de crime. 

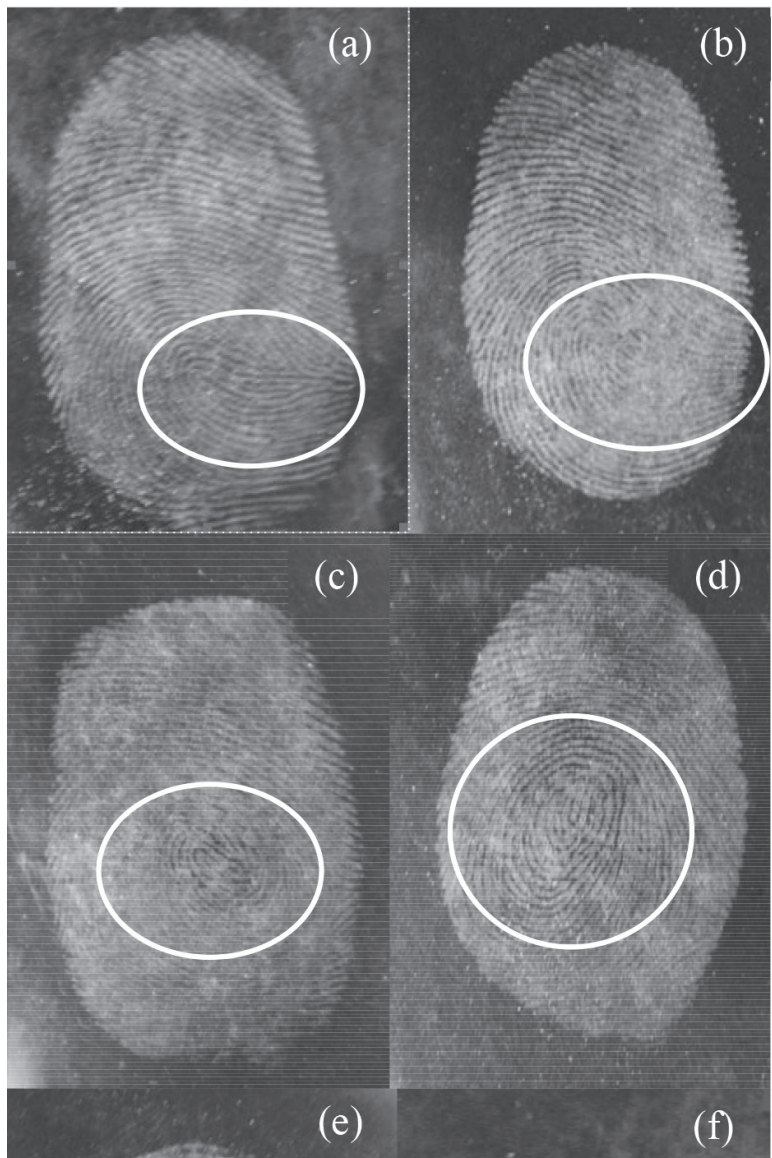

(e)
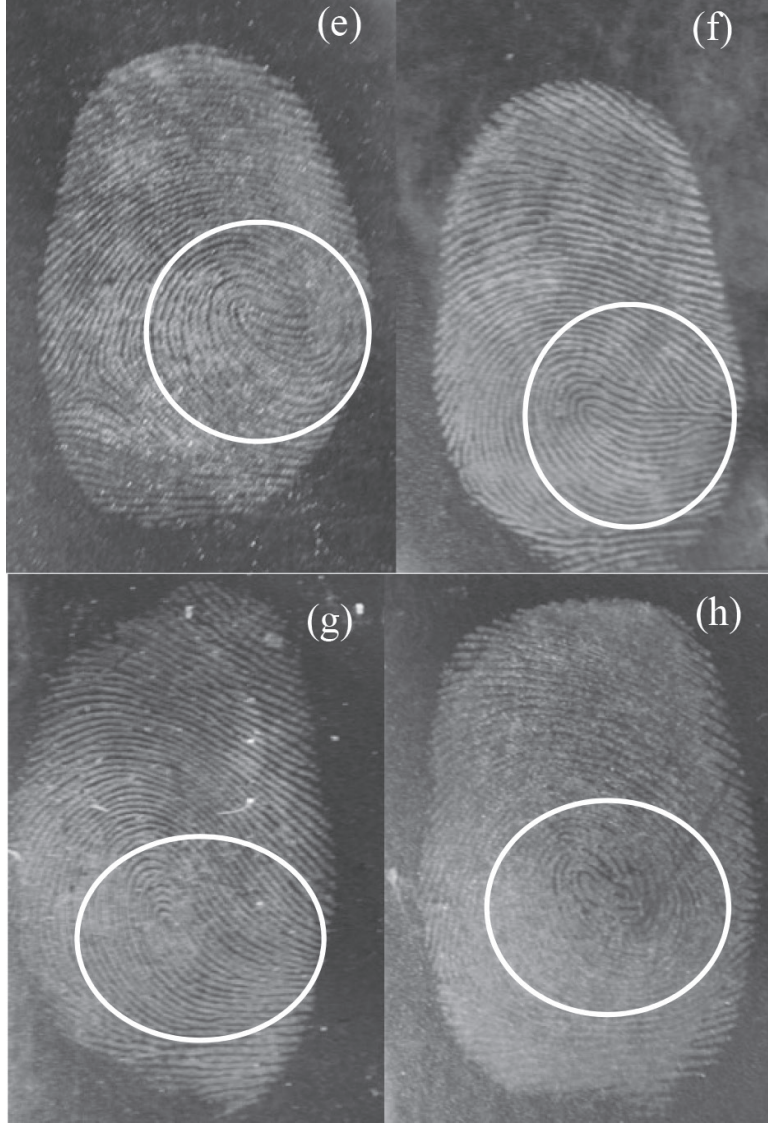

(h)

Figura 6. Imagem obtida com deposição sebácea de 8 doadores com a aplicação de chalcona sintetizada como revelador em pó. Classificação de acordo com o Sistema Vucetich de Classificação de Impressões Digitais (a) e (b): presilha extrerna; $(c)$ : presilha externa e (d): presilha interna; $(e)$ e $(f)$ : presilha externa; $(g)$ presilha externa e $(h)$ : verticilo
Como qualquer método, há limitações a considerar e baseia-se nos recursos avaliados na rotina pericial, mas os aspectos de evidência de impressão digital que demonstrem confiabilidade, dependem de cada caso pericial e, principalmente, da falta de comparação de uma metodologia padronizada com critérios definidos de revelação de impressão digital nos institutos periciais com a metodologia do estudo em questão.

\section{MATERIAL SUPLEMENTAR}

Informações adicionais sobre os resultados obtidos com RMN e cromatografia a gás acoplada à espectrometria de massas podem ser encontradas em http://quimicanova.sbq.org.br, em formato PDF, com acesso livre.

\section{AGRADECIMENTOS}

O presente trabalho foi realizado com apoio da Coordenação de Aperfeiçoamento de Pessoal de Nível Superior - Brasil (CAPES) - Código de Financiamento 001 CAPES. Os autores também agradecem ao Instituto Nacional de Ciência e Tecnologia (INCT Forensics - CNPq 465450 / 2014-8) pela assistência técnica e pelos recursos.

\section{REFERÊNCIAS}

1. Velho, J. A.; Geiser, G. C.; Espindula, A.; Ciências Forenses- Uma Introdução as principais áreas da criminalística moderna, $2^{\mathrm{a}}$ ed., Millennium: São Paulo, 2013.

2. Albrecht, J.; Biometrics, University of Pretoria: Pretória, 2003

3. http://www.biometricgroup.com/in_the_news, acessada em agosto 2019.

4. Bruni, T. A.; Velho, J. A.; Oliveira, M. F.; Fundamentos de Química Forense: Uma análise prática da química que soluciona crimes, $1^{\mathrm{a}} \mathrm{ed}$., Millenium: São Paulo, 2012.

5. http://www.quimica.net/emiliano/artigos/2006dez_forense1.pdf, acessada em agosto 2019.

6. http://tmp.mpce.mp.br/esmp/apresentacoes/I_Curso_de_Investigacao_ Criminal_Homic\%C3\%ADdio/02_Criminalistica_Geral_29_11_2012. pdf, acessada em agosto 2019.

7. Velho, J. A.; Costa, K. A.; Damasceno, C. T. M.; Locais de Crime: dos vestígios à dinâmica criminosa, $2^{\mathrm{a}}$ ed., Millenium: São Paulo, 2015.

8. Martins, B. S.; Oliveira, M. F.; Química Forense Experimental, $1^{\mathrm{a}}$ ed., Cengage Learning: São Paulo, 2016.

9. Castro, T. S.; Dissertação de mestrado, Universidade Federal de Juiz de Fora, Brasil, 2008.

10. Fergurson, S.; Nicholson, L.; Farrugia, R.; Bremer, D.; Guteh, D.; Sci. Justice 2013, 53, 67.

11. Figinni, A. R.; Silva, J. R.; Jobim, L. F.; Silva, M.; Identificação humana, $1^{\text {a }}$ ed., Millennium: São Paulo, 2003.

12. Champod, C.; Lennard, C.; Margot, P.; Stoilovic, M.; Fingerprints and Other Ridge Skin Impressions, $1^{\mathrm{a}}$ ed., CRC press: Florida, 2004.

13. Gireli, M. A.; Lobo, B. J. M.; Cunha, A. G.; Freitas, J. C. C.; Emmerich, F. G.; Sci. Int. 2015, 250, 26.

14. Almog, J.; Cantu, A.; Guidelines for the Assessment of Fingermark Detection Techniques, Journal of Forensic Identification 2014, 64, 174.

15. Fossoul, J. de A.; Pastris, C. M.; Feixat, C. B; Mcgrarr, L.; Brandelli, D.; Stow, K.; Badia, M. G.; J. Forensic Sci. 2016, 61, 12.

16. Pandhurnekar, C. P.; Meshram, E. M.; Himani, N.; Chopde, H. N.; Batra, R.; J. Org. Chem. Int. 2013, 1, 10.

17. Bano, S.; Javed, K.; Ahmad, S.; Rathish, I. G.; Singh, S.; Chaitanya, M.; Arunasree, K. M.; Alam, M. S.; Eur. J. Med. Chem. 2013, 65, 59.

18. Arancibia, R.; Biot, C.; Delaney, G.; Roussel, P.; Aurélie, Pascual, A.; Pradines, B.; Klahn, H.; J. Organomet. Chem. 2013, 723, 148. 
19. Monga, V.; Goyal, K.; Steindel, M.; Malhotra, M.; Rajani, D. P.; Rajani, S. D.; Med. Chem. Res. 2013, 23, 2019.

20. Vasconcelos, A.; Oliveira, P. S.; Ritter, M.; Freitag, R. A.; Romano, R. L.; Quina, F. H.; Pizzuti, L.; Pereira, C. M. P.; Stefanello, F. M.;
Barschak, A. G.; J. Biochem. Mol. Toxicol. 2011, 26, 161.

21. Balsan, J. D.; Santos, C. M. M.; Rosa, B. N.; Pacheco, B. S.; Pereira, C. M. P; Segatto, N. N.; Seixas, F. K ; Collares, T. V.; Passos, L. F.; Silva, C. C.; Mariotti, R. C.; BR 10201900238132019. 